\title{
Weighted Integral Inequalities with the Geometric Mean Operator
}

\author{
LARS-ERIK PERSSON $^{\mathrm{a}, *}$ and VLADIMIR D. STEPANOV ${ }^{\mathrm{b}, \dagger}$
}

a Department of Mathematics, Luleå University, S-97 187 Luleå, Sweden; ${ }^{b}$ Computer Centre, Russian Academy of Sciences, Far-Eastern Branch, Khabarovsk 680042, Russia

(Received 20 March 2001; Revised 29 June 2001)

The geometric mean operator is defined by

$$
G f(x)=\exp \left(\frac{1}{x} \int_{0}^{x} \log f(t) \mathrm{d} t\right)
$$

A precise two-sided estimate of the norm

$$
\|G\|=\sup _{f \neq 0} \frac{\|G f\|_{L_{u}^{q}}}{\|f\|_{L_{v}^{p}}}
$$

for $0<p, q \leq \infty$ is given and some applications and extensions are pointed out.

Keywords: Geometric mean operator; Hardy inequalities

Classification: AMS Subject Classification (1991): Primary: 26D15, 26D10; Secondary: $47 \mathrm{H} 19$

\footnotetext{
* Corresponding author. E-mail: larserik@sm.luth.se

† E-mail: stepanov@as.fe.ru
}

ISSN 1025-5834 print; ISSN 1029-242X. (C) 2002 Taylor \& Francis Ltd DOI: $10.1080 / 1025583021000022531$ 


\section{INTRODUCTION}

Applying the clever Polya's observation to the Hardy inequality, $p>1$

$$
\int_{0}^{\infty}\left(\frac{1}{x} \int_{0}^{x} f(t) \mathrm{d} t\right)^{p} \mathrm{~d} x \leq\left(\frac{p}{p-1}\right)^{p} \int_{0}^{\infty} f^{p}, \quad f \geq 0
$$

by changing $f \rightarrow f^{1 / p}$ and tending $p \rightarrow \infty$ we obtain the Knopp inequality [8] (c.f. also [2])

$$
\int_{0}^{\infty} G f(x) \mathrm{d} x \leq e \int_{0}^{\infty} f
$$

with the geometric mean operator

$$
G f(x):=\exp \left(\frac{1}{x} \int_{0}^{x} \log f(t) \mathrm{d} t\right), \quad f \geq 0 .
$$

The weighted integral inequality

$$
\left(\int_{0}^{\infty}(G f)^{q} u\right)^{1 / q} \leq \mathbb{C}\left(\int_{0}^{\infty} f^{p} v\right)^{1 / p}
$$

was investigated by several authors $[3-8,9,11,12]$ and a most general result was found by P. Gurka, B. Opic and L. Pick [11, 12] with, however, unstable constants pretending to estimate the norm (= the least possible constant $\mathbb{C}$ in (1)) (see (14) and (15) below).

In the present paper we give the precise two-sided estimate of the norm of $G: L_{v}^{p} \rightarrow L_{u}^{q}$ (see Theorems 2 and 4). In the case $0<p \leq q<\infty$ we argue close to the original Polya idea and for $0<q<p<\infty$ we use the Pick and Opic scheme [12] and a new form of the criterion for the Hardy inequality with weights (Theorem $3)$ which is of independent interest. Throughout the paper we denote $V(t)=\int_{0}^{t} v^{-1 /(p-1)}$ and undeterminates $0 \cdot \infty$ are taken to be equal to zero. 


\section{PICK AND OPIC SCHEME}

Put

$$
H f(x)=\frac{1}{x} \int_{0}^{x} f(t) \mathrm{d} t .
$$

It is well known that

$$
\lim _{\alpha \downarrow 0}\left(\frac{1}{x} \int_{0}^{x} f^{\alpha}\right)^{1 / \alpha}=G f(x)
$$

and

$$
\begin{aligned}
& \text { (i) } G f(x) \leq H f(x) \\
& \text { (ii) } G\left(f^{s}\right)=[G(f)]^{s}, \quad s \in \mathbb{R} \text {. }
\end{aligned}
$$

Let $0<p, q<\infty, u(x) \geq 0, v(x) \geq 0$ and put

$$
w:=\left[G\left(\frac{1}{v}\right)\right]^{q / p} u
$$

Then it follows from (3)(ii) that (1) $\Leftrightarrow$ (4) $\Leftrightarrow(5)$, where

$$
\begin{aligned}
\left(\int_{0}^{\infty}(G f)^{q} w\right)^{1 / q} & \leq \mathbb{C}\left(\int_{0}^{\infty} f^{p}\right)^{1 / p}, \\
\left(\int_{0}^{\infty}(G f)^{q s / p} w\right)^{p / q s} & \leq \mathbb{C}^{p / s}\left(\int_{0}^{\infty} f^{s}\right)^{1 / s}, \quad s>0 \\
\|G\|_{L_{v}^{p} \rightarrow L_{u}^{q}} & =\|G\|_{L^{p} \rightarrow L_{w}^{q}}=\|G\|_{L^{s} \rightarrow L_{w}^{s q / p}}^{s / p}
\end{aligned}
$$

and

$$
\|G\|_{X \rightarrow Y}:=\sup _{f \neq 0} \frac{\|G f\|_{Y}}{\|f\|_{X}} .
$$

It follows from Jensen's inequality (see (3)(i)), that $\|G\|_{X \rightarrow Y} \leq\|H\|_{X \rightarrow Y}$. Therefore the upper bounds for $\|G\|_{L_{v}^{p} \rightarrow L_{u}^{q}}$ can be derived from the following known estimates of $L^{p}-L_{w}^{q}$ norm of $\mathrm{H}$. 
(a) $1<p \leq q<\infty$. Then

$$
\mathbb{A} \leq\|H\|_{L^{p} \rightarrow L_{w}^{q}} \leq \alpha(p, q) \mathbb{A}
$$

where

$$
\mathbb{A}:=\mathbb{A}(p, q)=\sup _{t>0} \mathbb{A}(t)=\sup _{t>0}\left(\int_{t}^{\infty} \frac{w(x) \mathrm{d} x}{x^{q}}\right)^{1 / q} t^{1 / p^{\prime}},
$$

$\alpha(p, p)=p^{1 / p}\left(p^{\prime}\right)^{1 / p^{\prime}}$ and (Manakov [10])

$$
\begin{aligned}
& \alpha(p, q)=\left[\frac{\Gamma(q /(\lambda-1))}{\Gamma(\lambda / \lambda-1) \Gamma(q-1 / \lambda-1)}\right]^{(\lambda-1) / q}, \\
& 1<p<q<\infty, \quad \lambda=q / p .
\end{aligned}
$$

(b) $0<q<p \leq \infty, p>1,1 / r=1 / q-1 / p$. Then

$$
\beta_{1}(p, q) \mathbb{B} \leq\|H\|_{L^{p} \rightarrow L_{w}^{q}} \leq \beta_{2}(p, q) \mathbb{B}
$$

where

$$
\begin{gathered}
\mathbb{B}:=\mathbb{B}(p, q)=\left(\int_{0}^{\infty} t^{r / q^{\prime}}\left(\int_{t}^{\infty} \frac{w(x)}{x^{q}} \mathrm{~d} x\right)^{r / q} \mathrm{~d} t\right)^{1 / r}, \\
\beta_{1}(p, q)= \begin{cases}q^{1 / q}\left(p^{\prime}\right)^{1 / q^{\prime}} \frac{q}{r}, & 0<q<p<\infty, \quad p>1, \quad q \neq 1, \\
1, & 1=q<p<\infty, \quad 1 \leq q<p=\infty,\end{cases} \\
\beta_{2}(p, q)= \begin{cases}q^{1 / q}\left(p^{\prime}\right)^{1 / q^{\prime}}, & 1<q<p<\infty, \\
1, & 1=q<p<\infty, \quad 1 \leq q<p=\infty, \\
r^{1 / r} p^{1 / p}\left(p^{\prime}\right)^{1 / q^{\prime}}, & 0<q<1<p<\infty\end{cases}
\end{gathered}
$$

This implies the upper bound for $\|G\|$ in the case $p>1$. For the lower bound the following Lemma can be used. 
LEMMA 1 Let $0<p \leq q<\infty,\|G\|:=\|G\|_{L^{p} \rightarrow L_{w}^{q}}<\infty$. Then

$$
\begin{aligned}
& \|G\| \geq \sup _{t>0} t^{-1 / p}\left(\int_{0}^{t} w(x) \mathrm{d} x\right)^{1 / q}, \\
& \|G\| \geq \sup _{s>1}\left(\frac{(s-1) e^{s}}{1+(s-1) e^{s}}\right)^{1 / p} \sup _{t>0} t^{(s-1) / p}\left(\int_{t}^{\infty} \frac{w(x) \mathrm{d} x}{x^{s q / p}}\right)^{1 / q} .
\end{aligned}
$$

Proof We use a modified test function from the proof of ([5], Theorem 1.4). For $s>1, t>0$ put

$$
f(x)=t^{-1 / p} \chi_{[0, t]}(x)+(x e)^{-s / p} t^{(s-1) / p} \chi_{[t, \infty)}(x) .
$$

Then $\left(\int_{0}^{\infty} f^{p}\right)^{1 / p}=\left(1+\left((s-1) e^{s}\right)^{-1}\right)^{1 / p}=: a_{s}$ and (5) brings

$$
a_{s} \mathbb{C} \geq\left[t^{-q / p} \int_{0}^{t} w(x) \mathrm{d} x+t^{(s-1) q / p} \int_{t}^{\infty} \frac{w(x) \mathrm{d} x}{x^{s q / p}}\right]^{1 / q} .
$$

It gives (11) when $s \rightarrow \infty$ by omitting the second term on the right hand side and (12) by omitting the first term on the right hand side.

The lower bound for the case $0<q<p<\infty, p>1$ follows by putting the usual test function

$$
f(x)=x^{r /\left(p q^{\prime}\right)}\left(\int_{x}^{\infty} \frac{w(\tau) \mathrm{d} \tau}{\tau^{q}}\right)^{r /(p q)}
$$

in (4) ([12], Lemma 3.2). It brings

$$
\|G\| \geq e^{-r /\left(p q^{\prime}\right)}\left(\frac{q}{p^{\prime}}\right)^{1 / q} \mathbb{B} .
$$

Now, on the strength of (6) the upper bound from (7) and (12) imply the result of ([11], (1.3)): if $0<p \leq q<\infty$, then

$$
\begin{gathered}
\sup _{s>1}\left(\frac{(s-1) e^{s}}{1+(s-1) e^{s}}\right)^{1 / p} A^{s / p}\left(s, \frac{s q}{p}\right) \leq\|G\| \\
\leq \inf _{s>1} \alpha^{s / p}\left(s, \frac{s q}{p}\right) A^{s / p}\left(s, \frac{s q}{p}\right)
\end{gathered}
$$


with slightly better factors on both sides and the upper bound from (10) and (13) contains the result of ([12], (3.18)): if $0<q<p \leq \infty$, then

$$
\begin{gathered}
\sup _{s>1}\left(\frac{q(s-1)}{p}\right)^{1 / q} e^{(1-q s / p) /(p-q)} \mathbb{B}^{s / p}\left(s, \frac{q}{p} s\right) \leq\|G\| \\
\leq \inf _{s>1} \beta_{2}^{s / p}\left(s, \frac{s q}{p}\right) \mathbb{B}^{s / p}\left(s, \frac{q}{p} s\right)
\end{gathered}
$$

with

$$
\mathbb{A}^{s / p}\left(s, \frac{s q}{p}\right)=\sup _{t>0} t^{(s-1) / p}\left(\int_{t}^{\infty} \frac{w(x) \mathrm{d} x}{x^{s q / p}}\right)^{1 / q}
$$

and

$$
\mathbb{B}^{s / p}\left(s, \frac{s q}{p}\right)=\left(\int_{0}^{\infty} t^{(q s-p) /(p-q)}\left(\int_{t}^{\infty} \frac{w(x) \mathrm{d} x}{x^{s q / p}}\right)^{s /(p-q)} \mathrm{d} t\right)^{1 / r} .
$$

\section{THE CASE $0<\boldsymbol{p} \leq \boldsymbol{q}<\infty$}

We are going to use a limiting consideration originally due to G. Polya. To this end we replace (4) by

$$
\left(\int_{0}^{\infty}\left(H f^{\alpha}\right)^{q / \alpha} w\right)^{1 / q} \leq \mathbb{C}_{\alpha}\left(\int_{0}^{\infty} f^{p}\right)^{1 / p}, \quad \alpha>0
$$

which is equivalent to the weighted Hardy inequality

$$
\left(\int_{0}^{\infty}(H f)^{q / \alpha} w\right)^{\alpha / q} \leq \mathbb{C}_{\alpha}^{\alpha}\left(\int_{0}^{\infty} f^{p / \alpha}\right)^{\alpha / p}, \quad \alpha>0
$$

and using (3) we reduce the problem to existence of the limits of upper and lower bounds for the norm $\|H\|_{L^{p / \alpha} \rightarrow L_{w / \alpha}^{q / \alpha}}^{1 / \alpha}$ because

$$
\|G\|_{L_{v}^{p} \rightarrow L_{u}^{q}}=\lim _{\alpha \downarrow 0}\|H\|_{L^{p / \alpha} \rightarrow L_{w}^{q / \alpha}}^{1 / \alpha}
$$

To this purpose we need the following alternate criterion for the weighted Hardy inequality ([14], Section 2.3). 
TheOREM 1 Let $1<p \leq q<\infty$. Then

$$
\left(\int_{0}^{\infty}\left(\int_{0}^{x} f\right)^{q} u(x) \mathrm{d} x\right)^{1 / q} \leq \mathbb{C}\left(\int_{0}^{\infty} f^{p} v\right)^{1 / p}
$$

is true for all $f \geq 0$ iff

$$
\infty>\mathcal{A}_{1}=\sup _{t>0} V^{-1 / p}(t)\left(\int_{0}^{t} u(x) V^{q}(x) \mathrm{d} x\right)^{1 / q}
$$

and

$$
\mathcal{A}_{1} \leq \mathbb{C} \leq p^{\prime} \mathcal{A}_{1}
$$

Proof With $p^{\prime}=p /(p-1), q^{\prime}=q /(q-1)$ inequality (17) is equivalent to

$$
\left(\int_{0}^{\infty}\left(\int_{x}^{\infty} g\right)^{p^{\prime}} \mathrm{d} V(x)\right)^{1 / p^{\prime}} \leq \mathbb{C}\left(\int_{0}^{\infty} g^{q^{\prime}} u^{-1 /(q-1)}\right)^{1 / q^{\prime}}
$$

with the same constant $\mathbb{C}$. We have for $g$ with supp $g \subset(0, \infty)$

$$
\begin{aligned}
J & :=\int_{0}^{\infty}\left(\int_{x}^{\infty} g\right)^{p^{\prime}} \mathrm{d} V(x)=p^{\prime} \int_{0}^{\infty}\left(\int_{x}^{\infty} g\right)^{1 /(p-1)} g(x) V(x) \mathrm{d} x \\
& \leq p^{\prime}\left(\int_{0}^{\infty} g^{q^{\prime}} u^{-1 /(q-1)}\right)^{1 / q^{\prime}}\left(\int_{0}^{\infty}\left(\int_{x}^{\infty} g\right)^{q /(p-1)} u(x) V^{q}(x) \mathrm{d} x\right)^{1 / q} \\
& :=p^{\prime}\left(\int_{0}^{\infty} g^{q^{\prime}} u^{-1 /(q-1)}\right)^{1 / q^{\prime}} J_{1}^{1 / q} .
\end{aligned}
$$

Now

$$
\begin{aligned}
J_{1} & =\int_{0}^{\infty} \int_{x}^{\infty} \mathrm{d}\left(-\left(\int_{t}^{\infty} g\right)^{q /(p-1)}\right) u(x) V^{q}(x) \mathrm{d} x \\
& =\int_{0}^{\infty}\left[\mathrm{d}\left(-\left(\int_{t}^{\infty} g\right)^{q /(p-1)}\right)\right] \int_{0}^{t} u(x) V^{q}(x) \mathrm{d} x
\end{aligned}
$$


(applying (18) and Minkowski’s inequality)

$$
\begin{aligned}
& \leq \mathcal{A}_{1}^{q} \int_{0}^{\infty}\left[\mathrm{d}\left(-\left(\int_{t}^{\infty} g\right)^{q /(p-1)}\right)\right] V^{q / p}(t) \\
& \leq \mathcal{A}_{1}^{q}\left(\int_{0}^{\infty}\left(\int_{x}^{\infty}\left[\mathrm{d}\left(-\left(\int_{t}^{\infty} g\right)^{q /(p-1)}\right)\right]\right)^{p / q} \mathrm{~d} V(x)\right)^{q / p} \\
& =\mathcal{A}_{1}^{q}\left(\int_{0}^{\infty}\left(\int_{x}^{\infty} g\right)^{p^{\prime}} \mathrm{d} V(x)\right)^{q / p} .
\end{aligned}
$$

Thus, $\quad J^{1 / p^{\prime}} \leq p^{\prime} \mathcal{A}_{1}\left(\int_{0}^{\infty} g^{q^{\prime}} u^{-1 /(q-1)}\right)^{1 / q^{\prime}} \Rightarrow \mathbb{C} \leq p^{\prime} \mathcal{A}_{1}$. Putting $f_{t}=$ $\chi_{[0, t]} v^{-1 /(p-1)}$ in (17) we obtain $\mathcal{A}_{1} \leq \mathbb{C}$.

THEOREM 2 Let $0<p \leq q<\infty$. Then the inequality (1) holds for all $f \geq 0$ iff

$$
\mathbb{D}:=\sup _{t>0} t^{-1 / p}\left(\int_{0}^{t} w(x) \mathrm{d} x\right)^{1 / q}<\infty
$$

and

$$
\mathbb{D} \leq\|G\|_{L_{v}^{p} \rightarrow L_{u}^{q}} \leq e^{1 / p} \mathbb{D} .
$$

Proof It follows from Theorem 1, that for $0<\alpha<p \leq q<\infty$

$$
\mathbb{D} \leq\|H\|_{L^{p / \alpha} \rightarrow L_{w}^{q / x}}^{1 / \alpha} \leq\left(\frac{p}{p-\alpha}\right)^{1 / \alpha} \mathbb{D}
$$

and (20) is a consequence of (16). The lower bound in (20) was also proved in Lemma 1 (11).

Remark 1 The factor $e^{1 / p}$ is the hest possible for $p=q$ and attains in the case $u(x)=v(x)=1$. For $p=q=1$ an alternate form of Theorem 2 was proved in ([5], Theorem 1.4). The factor $p^{\prime}$ in (19) is best possible for only $p=q$. When $1<p<q<\infty$ it can be improved in general according to the following Lemma. 
LEMMA 2 Suppose $1<p<q<\infty$ and $V(\infty)=\infty$. Then there exists a weight $u^{*}(x) \geq 0$ such that $\mathcal{A}_{1}=1$ and

$$
\left(\int_{0}^{\infty}\left(\int_{0}^{x} f\right)^{q} u^{*}(x) \mathrm{d} x\right)^{1 / q} \leq \alpha^{*}(p, q)\left(\int_{0}^{\infty} f^{p} v\right)^{1 / p}
$$

where $p^{\prime}>\alpha^{*}(p, q)$ and $\alpha^{*}(p, q)$ is given by

$$
\alpha^{*}(p, q)=(p-1)^{-1 / q}\left[\frac{\Gamma(q p /(q-p))}{\Gamma(q /(q-p)) \Gamma((q-1) p /(q-p))}\right]^{(q-p) / q p} .
$$

Proof Let

$$
V^{-1 / p}(t)\left(\int_{0}^{t} u^{*}(x) V^{q}(x) \mathrm{d} x\right)^{1 / q} \equiv 1, \quad t>0 .
$$

Then

$$
u^{*}=\frac{q}{p} V^{-q / p^{\prime}-1} v^{-1 /(p-1)} .
$$

Using the change of variables

$$
V(t)=s, f(t) v^{1 /(p-1)}(t)=g(s)
$$

we find

$$
\int_{0}^{\infty} f^{p} v=\int_{0}^{\infty}\left[f(t) v^{1 /(p-1)}(t)\right]^{p} \mathrm{~d} V(t)=\int_{0}^{\infty} g^{p}
$$

and

$$
\begin{aligned}
\int_{0}^{\infty}\left(\int_{0}^{x} f\right)^{q} u^{*}(x) \mathrm{d} x & =\frac{q}{p} \int_{0}^{\infty}\left(\int_{0}^{x} f(t) v^{1 /(p-1)}(t) \mathrm{d} V(t)\right)^{q} V^{-q / p^{\prime}-1}(x) \mathrm{d} V(x) \\
& =\frac{q}{p} \int_{0}^{\infty}\left(\int_{0}^{y} g\right)^{q} y^{-q / p^{\prime}-1} \mathrm{~d} y .
\end{aligned}
$$

Thus, inequality (17) with $v$ and $u^{*}$ satisfying (21) becomes

$$
\left(\int_{0}^{\infty}\left(\int_{0}^{y} g\right)^{q} y^{-q / p^{\prime}-1} \mathrm{~d} y\right)^{1 / q} \leq\left(\frac{p}{q}\right)^{1 / q} \mathbb{C}\left(\int_{0}^{\infty} g^{p}\right)^{1 / p}
$$


and by the result of G. A. Bliss [1] we conclude that

$$
\left(\frac{p}{q}\right)^{1 / q} \mathbb{C}=C_{p, q}
$$

where

$$
\begin{aligned}
C_{p, q}= & \left(\frac{q}{p}-1\right)^{-1 / q}\left(\frac{q(p-1)}{q-p}\right)^{-1 / p} \\
& \times\left[\frac{\Gamma(q p /(q-p))}{\Gamma(q /(q-p)) \Gamma(q(p-1) /(q-p))}\right]^{(q-p) / p q}
\end{aligned}
$$

and the result follows.

Now, it makes sense to look what the limiting procedure gives if it starts from (7-9).

Proposition 1 Let $0<p \leq q<\infty$. Then the following upper bound holds

$$
\|G\|_{L_{r}^{p} \rightarrow L_{u}^{q}} \leq \gamma(p, q) \sup _{t>0} t^{1 / q-1 / p} w^{1 / q}(t),
$$

where $\gamma(p, p)=e^{1 / p}$ and

$$
\gamma(p, q)=\left[\left(\frac{q}{p}-1\right)\left(\Gamma\left(\frac{q}{q-p}\right)\right)^{(q / p)-1}\right]^{-1 / q}<e^{1 / q}, \quad p<q .
$$

Proof Since $\lim _{q \downarrow p} \gamma(p, q)=e^{1 / p}$, we consider the case $p<q$ only. Using (7) and (9) we find for $0<\alpha<p \leq q$

$$
\|H\|_{L^{\prime / \alpha} \rightarrow L_{*}^{q / \alpha}}^{1 / \alpha} \leq\left[\frac{\Gamma(q /(\lambda-1) \alpha)}{\Gamma(\lambda /(\lambda-1)) \Gamma(((q / \alpha)-1) /(\lambda-1))}\right]^{(\lambda-1) / q} \mathbb{A}_{\alpha},
$$

where

$$
\mathbb{A}_{\alpha}=\sup _{t>0} t^{1 / \alpha-1 / p}\left(\int_{t}^{\infty} \frac{w(x) \mathrm{d} x}{x^{q / \alpha}}\right)^{1 / q}
$$


Denote $q / \alpha(\lambda-1)=x$. It is known that

$$
\begin{aligned}
& \frac{\Gamma(q /(\lambda-1) \alpha)}{\Gamma(q /(\lambda-1) \alpha-1 /(\lambda-1))(q /(\lambda-1) \alpha)^{1 /(\lambda-1)}} \\
& \quad=\frac{\Gamma(x)}{\Gamma(x-1 /(\lambda-1)) x^{1 /(\lambda-1)}} \rightarrow 1, \quad x \rightarrow \infty .
\end{aligned}
$$

Hence, (16) yields

$$
\|G\|_{L_{v}^{p} \rightarrow L_{u}^{q}} \leq \gamma(p, q) \sup _{t>0} \mathbb{A}_{0}(t)
$$

where

$$
\mathbb{A}_{0}(t)=\limsup _{\alpha \downarrow 0}\left(\frac{q}{\alpha} t^{-q / p} \int_{t}^{\infty} \frac{w(x) \mathrm{d} x}{(x / t)^{q / \alpha}}\right)^{1 / q} .
$$

Denoting $q / \alpha=s \uparrow \infty$ when $\alpha \downarrow 0$ we observe that

$$
\mathbb{A}_{0}(t)=t^{1 / q-1 / p} \limsup _{s \uparrow \infty}\left(\frac{s-1}{t} \int_{t}^{\infty} \frac{w(x) \mathrm{d} x}{(x / t)^{s}}\right)^{1 / q} .
$$

Without loss of generality we suppose that $w(x)$ is a step function, and note that

$$
\chi_{[1, \infty)}(x)(s-1) x^{-s} \rightarrow \delta_{1}(x), \quad s \uparrow \infty,
$$

where $\delta_{1}(x)$ is the Dirac delta function with the unit mass at $x=1$. Then

$$
\mathbb{A}_{0}(t)=t^{1 / q-1 / p} w(t) \text { a.e. } t>0
$$

and (22) follows. Finally we prove that

$$
\gamma(p, q)<e^{1 / q}, \quad p<q .
$$

Indeed, this is equivalent to

$$
g(\lambda):=(\lambda-1) \Gamma^{\lambda-1}\left(\frac{\lambda}{\lambda-1}\right)>e^{-1} .
$$


We have

$$
\begin{aligned}
g(\lambda) & =\left(\int_{0}^{\infty}(x(\lambda-1))^{1 /(\lambda-1)} e^{-x} \mathrm{~d} x\right)^{\lambda-1} \\
& =\left(\int_{0}^{\infty} t^{1 /(\lambda-1)} e^{-t /(\lambda-1)} \frac{\mathrm{d} t}{\lambda-1}\right)^{\lambda-1} \\
& =\left(1-\frac{1}{\lambda-1} \int_{0}^{\infty}\left(1-t^{1 /(\lambda-1)}\right) e^{-t /(\lambda-1)} \mathrm{d} t\right)^{\lambda-1}
\end{aligned}
$$

Plainly

$$
\int_{0}^{\infty}\left(1-t^{1 /(\lambda-1)}\right) e^{-t /(\lambda-1)} \mathrm{d} t<\int_{0}^{1}\left(1-t^{1 /(\lambda-1)}\right) e^{-t /(\lambda-1)} \mathrm{d} t<1 .
$$

Thus,

$$
g(\lambda)>\left(1-\frac{1}{\lambda-1}\right)^{\lambda-1}>e^{-1}
$$

\section{THE CASE $\boldsymbol{0}<\boldsymbol{q}<\boldsymbol{p} \leq \infty$}

The aim of this section is to find a criterion for the inequality (1) similar to (20) in the opposite case of relation between parameters $p$ and $q$. It means that we want to replace the Pick and Opic result (15) by a two-sided estimate with stable factor as in (20). For this purpose we need a new criterion for the weighted Hardy inequality in the case $q<p$.

THEOREM 3 Let $0<q<p<\infty, p>1,1 / r=1 / q-1 / p$. Then (17) is true for all $f \geq 0$ iff

$$
\mathcal{B}:=\left(\int_{0}^{\infty}\left(\int_{0}^{t} u V^{q}\right)^{r / p} u(t) V^{q-r / p}(t) \mathrm{d} t\right)^{1 / r}<\infty
$$

Moreover, if $V(\infty)=\infty$, then

$$
\left(p^{\prime}\right)^{1 / q^{\prime}}(q / r)^{1 / r^{\prime}} 2^{-1 / q} \mathcal{B} \leq C \leq q^{1 / p} p^{1 / r} p^{\prime} \mathcal{B}
$$


and if $0<V(\infty)<\infty$, then

$$
\begin{array}{r}
{\left[\frac{q}{r}+2^{r / q} r^{r-1} q^{-2 r / p}\left(q p^{\prime}\right)^{-r / q^{\prime}}\right]^{-1 / r} \mathcal{B} \leq C} \\
\leq\left(\frac{r}{q}\right)^{1 / r}\left[4^{q}+q\left(p^{\prime}\right)^{q}\left(\frac{p}{r}\right)^{q / r}\right]^{1 / q} \mathcal{B}
\end{array}
$$

Proof Suppose that $V(\infty)=\infty$. Then

$$
\mathcal{B}=\left(\frac{q}{p}\right)^{1 / r}\left(\int_{0}^{\infty}\left(\int_{0}^{t} u V^{q}\right)^{r / q} V^{-r / q}(t) \mathrm{d} V(t)\right)^{1 / r}=:\left(\frac{q}{p}\right)^{1 / r} \mathcal{B}_{0}
$$

Indeed, if $\mathcal{B}<\infty$, then

$$
\begin{aligned}
& \left(\int_{0}^{t} u V^{q}\right)^{r / q} V^{-r / p}(t)=V^{-r / p}(t) \int_{0}^{t} \mathrm{~d}\left(\int_{0}^{x} u V^{q}\right)^{r / q} \\
& \quad \leq \frac{r}{q} \int_{0}^{t}\left(\int_{0}^{x} u V^{q}\right)^{r / p} u(x) V^{q-r / p}(x) \mathrm{d} x \rightarrow 0, \quad t \rightarrow 0 .
\end{aligned}
$$

Integrating by parts we find that $\mathcal{B} \geq(q / p)^{1 / r} \mathcal{B}_{0}$. Hence, $\mathcal{B}_{0}<\infty$ and

$$
\begin{aligned}
& \left(\int_{0}^{t} u V^{q}\right)^{r / q} V^{-r / p}(t)=\left(\int_{0}^{t} u V^{q}\right)^{r / q} \int_{t}^{\infty} \mathrm{d}\left(-V^{-r / p}(x)\right) \\
& \leq \frac{r}{p} \int_{t}^{\infty}\left(\int_{0}^{x} u V^{q}\right)^{r / q} V^{-r / q}(x) \mathrm{d} V(x) \rightarrow 0, \quad t \rightarrow \infty
\end{aligned}
$$

Again, integrating by parts, we see that $\mathcal{B}_{0} \geq(p / q)^{1 / r} \mathcal{B}$. Consequently, $\mathcal{B}=(q / p)^{1 / r} \mathcal{B}_{0}$. The samie arguments work if we start with $\mathcal{B}_{0}<\infty$.

Observe, that if $0<V(\infty)<\infty$, then

$$
\mathcal{B}^{r}=\frac{q}{r} V^{-r / p}(\infty)\left(\int_{0}^{\infty} u V^{q}\right)^{r / q}+\frac{q}{p} \mathcal{B}_{0}^{r}
$$

For the lower bound we suppose that inequality (17) holds with $\mathbb{C}<\infty$. Then according to [13]

$$
\mathbb{C} \geq q^{1 / q}\left(p^{\prime}\right)^{1 / q^{\prime}} \frac{q}{r} B
$$


where

$$
B=\left(\int_{0}^{\infty}\left(\int_{x}^{\infty} u\right)^{r / q} V^{r / q^{\prime}}(x) \mathrm{d} V\right)^{1 / r}
$$

We show that

$$
\mathcal{B}_{0} \leq(2 q)^{1 / q}(p / r)^{1 / r}
$$

and the lower bound (23) will be proved. By writing

$$
\mathcal{B}_{0}^{r}=\int_{0}^{\infty}\left(\int_{0}^{x} V^{q}(t) \mathrm{d}\left(-\int_{t}^{x} u\right)\right)^{r / q} V^{-r / q}(x) \mathrm{d} V(x)
$$

we find

$$
\begin{aligned}
\int_{0}^{x} V^{q}(t) \mathrm{d}\left(-\int_{t}^{x} u\right) & =q \int_{0}^{x}\left(\int_{t}^{x} u\right) V^{q-1}(t) \mathrm{d} V(t) \\
& =q \int_{0}^{x}\left\{\left(\int_{t}^{x} u\right) V^{q-1+q / 2 p}(t)\right\} V^{-q / 2 p}(t) \mathrm{d} V(t)
\end{aligned}
$$

applying Hölder's inequality with the exponents $r / q$ and $p / q$

$$
\leq q\left(\int_{0}^{x}\left(\int_{t}^{x} u\right)^{r / q} V^{(q-1+q / 2 p) r / q} \mathrm{~d} V\right)^{q / r}\left(\int_{0}^{x} V^{-1 / 2} \mathrm{~d} V\right)^{q / p} .
$$

This and (28) imply

$$
\begin{aligned}
\mathcal{B}_{0}^{r} & \leq q^{r / q} 2^{r / p} \int_{0}^{\infty}\left(\int_{0}^{x}\left(\int_{t}^{\infty} u\right)^{r / q} V^{(q-1+q / 2 p) r / q} \mathrm{~d} V(t)\right) V^{r / 2 p-r / q}(x) \mathrm{d} V(x) \\
& =q^{r / p} 2^{r / p} \int_{0}^{\infty}\left(\int_{t}^{\infty} u\right)^{r / q} V^{r / q^{\prime}+r / 2 p}(t) \mathrm{d} V(t) \int_{t}^{\infty} V^{r / 2 p-r / q}(x) \mathrm{d} V(x) \\
& \leq \frac{(2 q)^{r / q} p}{r} \int_{0}^{\infty}\left(\int_{t}^{\infty} u\right)^{r / q} V^{r / q^{\prime}}(t) \mathrm{d} V(t)
\end{aligned}
$$

and (27) follows, which together with (26) gives the lower bound of (23). 
For the upper bound we assume first that $\mathcal{B}<\infty$ and $V(\infty)=\infty$. Then

$$
\begin{aligned}
J & :=\int_{0}^{\infty}\left(\int_{0}^{x} f\right)^{q} u(x) \mathrm{d} x=\int_{0}^{\infty}\left(\int_{0}^{x} f\right)^{q} u(x) V^{q}(x) V^{-q}(x) \mathrm{d} x \\
& =q \int_{0}^{\infty}\left(\int_{0}^{x} f\right)^{q} u(x) V^{q}(x) \int_{x}^{\infty} V^{-q-1}(s) \mathrm{d} V(s) \mathrm{d} x=: J_{0} \\
& =q \int_{0}^{\infty} V^{-q-1}(s)\left(\int_{0}^{s}\left(\int_{0}^{x} f\right)^{q} u(x) V^{q}(x) \mathrm{d} x\right) \mathrm{d} V(s) \\
& \leq q \int_{0}^{\infty}\left\{\left(\int_{0}^{s} f\right)^{q} V^{-q}(s)\right\}\left\{\left(\int_{0}^{s} u V^{q}\right) V^{-1}(s)\right\} \mathrm{d} V(s)
\end{aligned}
$$

(applying Hölder's inequality with the exponents $p / q$ and $r / q$ )

$$
\leq q\left(\int_{0}^{\infty}\left(\int_{0}^{s} f\right)^{p} \frac{\mathrm{d} V(s)}{V^{p}(s)}\right)^{q / p} \mathcal{B}_{0}^{q}
$$

It is easy to see, that by Theorem 1

$$
\left(\int_{0}^{\infty}\left(\int_{0}^{s} f\right)^{p} \frac{\mathrm{d} V(s)}{V^{p}(s)}\right)^{1 / p} \leq p^{\prime}\left(\int_{0}^{\infty} f^{p} v\right)^{1 / p},
$$

and the upper bound in (23) follows.

Now, let $0<V(\infty)<\infty$ and $\mathcal{B}<\infty$. Arguing as above we find

$$
J=J_{0}+V^{-q}(\infty) \int_{0}^{\infty}\left(\int_{0}^{x} f\right)^{q} u(x) V^{q}(x) \mathrm{d} x=: J_{0}+J_{1} .
$$

We need to estimate $J_{1}$. To this end let $\left\{x_{k}\right\} \subset(0, \infty), k \leq N<\infty$ be such a sequence, that

$$
\begin{gathered}
\int_{0}^{x_{k}} f=2^{k}, \quad k \leq N, \\
\int_{0}^{\infty} f \leq 2^{N+1}, \quad x_{N+1}=\infty .
\end{gathered}
$$


The last is possible because

$$
\int_{0}^{\infty} f \leq\left(\int_{0}^{\infty} f^{p} v\right)^{1 / p} V^{1 / p^{\prime}}(\infty)<\infty
$$

Thus,

$$
\begin{aligned}
V^{q}(\infty) J_{1} & =\sum_{k \leq N} \int_{x_{k}}^{x_{k+1}}\left(\int_{0}^{x} f\right)^{q} u(x) V^{q}(x) \mathrm{d} x \leq \sum_{k \leq N} 2^{(k+1) q} \int_{x_{k}}^{x_{k+1}} u V^{q} \\
& \leq 4^{q} \sum_{k \leq N}\left(\int_{x_{k-1}}^{x_{k}} f^{p} v\right)^{q / p}\left(\int_{x_{k-1}}^{x_{k}} v^{-1 /(p-1)}\right)^{q / p^{\prime}} \int_{x_{k}}^{x_{k+1}} u V^{q} \\
& \leq 4^{q}\left(\int_{0}^{\infty} f^{p} v\right)^{q / p}\left(\sum_{k \leq N} V^{r / p^{\prime}}\left(x_{k}\right)\left(\int_{x_{k}}^{x_{k+1}} u V^{q}\right)^{r / q}\right)^{q / r} \\
& \leq\left(\frac{r}{q}\right)^{q / r} 4^{q}\left(\int_{0}^{\infty} f^{p} v\right)^{q / p}\left(\int_{0}^{\infty}\left(\int_{0}^{x} u V^{q}\right)^{r / p} u(x) V^{q+r / p^{\prime}}(x) \mathrm{d} x\right)^{q / r} \\
& \leq\left(\frac{r}{q}\right)^{q / r} 4^{q} V^{q}(\infty)\left(\int_{0}^{\infty} f^{p} v\right)^{q / p} \mathcal{B}^{q} .
\end{aligned}
$$

Therefore,

$$
J_{1}^{1 / q} \leq\left(\frac{r}{q}\right)^{1 / r}\left[q\left(p^{\prime}\right)^{q}\left(\frac{p}{r}\right)^{q / r}+4^{q}\right]^{1 / q} \mathcal{B}\left(\int_{0}^{\infty} f^{p} v\right)^{1 / p}
$$

and the upper bound in (24) is proved. For the lower bound we note that (17) with $f=v^{-1 /(p-1)}$ brings

$$
C \geq V^{-1 / p}(\infty)\left(\int_{0}^{\infty} u V^{q}\right)^{1 / q}
$$

Now, combining this with (25-27), we obtain the left hand side of (24). 
The main result of this section is the following.

THEOREM 4 Let $0<q<p<\infty, 1 / r=1 / q-1 / p$. Then

$$
\|G\|_{L_{v}^{p} \rightarrow L_{u}^{q}} \approx\left(\int_{0}^{\infty}\left(\frac{1}{x} \int_{0}^{x} w\right)^{r / p} w(x) \mathrm{d} x\right)^{1 / r}
$$

with factors of equivalence depending on $p$ and $q$ only.

Proof It follows from (6), (10) and (15) that

$$
\|G\|_{L_{v}^{p} \rightarrow L_{u}^{q}} \approx\|H\|_{L^{s} \rightarrow L_{w}^{s q / p}}^{s / p}, \quad s>1
$$

and from the case $V(\infty)=\infty$ of Theorem 3 we know that

$$
\|H\|_{L^{s} \rightarrow L_{w}^{s / p}}^{s / p} \approx\left(\int_{0}^{\infty}\left(\frac{1}{x} \int_{0}^{x} w\right)^{r / p} w(x) \mathrm{d} x\right)^{1 / r}:=\mathcal{B}_{w}
$$

with factors depending on $p, q$ and $s>1$ but not $w$. More precisely,

$$
\gamma_{1}(p, q) B_{w} \leq\|G\|_{L_{v}^{p} \rightarrow L_{u}^{q}} \leq \gamma_{2}(p, q) B_{w},
$$

where

$$
\begin{aligned}
& \gamma_{1}(p, q)=2^{-1 / q}\left(\frac{q}{p}\right)^{1 / p}\left(1-\frac{q}{p}\right)^{-1 / r} \sup _{s>1} s^{1 / p}\left(\frac{s}{s-1}\right)^{s / p-1 / q}\left(1-\frac{q}{p}\right)^{s / p}, \\
& \gamma_{2}(p, q)=\left(\frac{q}{p}\right)^{1 / p} \inf _{s>1} s^{1 / q}\left(\frac{s}{s-1}\right)^{s / p} .
\end{aligned}
$$

\section{CONCLUDING REMARKS}

The results obtained in this paper can be formulated in a more general way. Here we just as an example study the operators

$$
G_{a} f(x)=\exp \left(\frac{a}{x^{a}} \int_{0}^{x} t^{a-1} \ln |f(t)| \mathrm{d} t\right), \quad a>0,
$$


and put

$$
u_{a}(x)=\frac{1}{a} x^{1 / a-1} u\left(x^{1 / a}\right), \quad v_{a}=\frac{1}{a} x^{1 / a-1} v\left(x^{1 / a}\right)
$$

and

$$
w_{a}=\left[G_{a}\left(\frac{1}{v_{a}}\right)\right]^{q / p} u_{a}
$$

Then Theorem 2 can (formally) be generalized as follows:

TheOREM 5 Let $0<p \leq q<\infty$. Then the inequality

$$
\left(\int_{0}^{\infty}\left(G_{a} f\right)^{q} u\right)^{1 / q} \leq C_{a}\left(\int_{0}^{\infty} f^{p} v\right)^{1 / p}, \quad f \geq 0
$$

is valid if and only if

$$
D_{a}:=\sup _{t>0} t^{-1 / p}\left(\int_{0}^{t} w_{a}(x) \mathrm{d} x\right)^{1 / q}<\infty
$$

and

$$
D_{a} \leq\left\|G_{a}\right\|_{L_{v}^{p} \rightarrow L_{u}^{q}} \leq e^{1 / p} D_{a} .
$$

Proof Note that

$$
G_{a} f(x)=\frac{1}{x^{a}} \int_{0}^{x} \ln f(t) \mathrm{d} t^{a},
$$

make the variable transformation $y=t^{a}$ and after that $z=x^{a}$ in (29) and the result follows from Theorem 2.

In particular, by applying Theorem 5 with $v(t)=t^{\beta}$ and $u(t)=t^{\alpha}$ we obtain:

Example Let $\alpha, \beta \in \mathbb{R}, a>0$ and $0<p \leq q<\infty$. Then the inequality

$$
\left(\int_{0}^{\infty} x^{\alpha}\left(\exp a x^{-a} \int_{0}^{x} x^{a-1} \ln f(t) \mathrm{d} t\right)^{q}\right)^{1 / q} \leq C\left(\int_{0}^{\infty}(f(x))^{p} x^{\beta} \mathrm{d} x\right)^{1 / p}
$$


for some finite $C>0$ iff

$$
\left(\frac{1+\alpha}{a}\right) \frac{1}{q}-\left(\frac{1+\beta}{a}\right) \frac{1}{p}=0 .
$$

Moreover

$$
C \leq a^{1 / q-1 / p} e^{((1+\beta) / a p)}\left(\left(1-\frac{1+\beta}{a}\right) \frac{q}{p}+\frac{(1+\alpha)}{a}\right)^{1 / q} .
$$

In particular, for the case $p=q=1, \beta=\alpha$ we obtain the following well-known inequality by Cochran and Lee ([3], Theorem 1):

$$
\int_{0}^{\infty} x^{\alpha} \exp \left(a x^{-a} \int_{0}^{x} x^{a-1} \ln f(t) \mathrm{d} t\right) \mathrm{d} x \leq e^{(\alpha+1) / a} \int_{0}^{\infty} x^{\alpha} f(x) \mathrm{d} x .
$$

c.f. also [4].

In the same way Theorem 4 can be generalized in the following way:

THEOREM 6 Let $0<q<p<\infty, 1 / r=1 / q-1 / p$. Then the inequality (29) holds for all $f \geq 0$ iff

$$
C_{a}=\left(\int_{0}^{\infty}\left(\frac{1}{x} \int_{0}^{x} w_{a}\right)^{r / p} w_{a}(x) \mathrm{d} x\right)^{1 / r}<\infty
$$

and

$$
\left\|G_{a}\right\|_{L_{v}^{p} \rightarrow L_{u}^{q}} \approx C_{a}
$$

\section{Acknowledgements}

Vladimir D. Stepanov was partially supported by the RFBR grant $00-$ 01-00239.

\section{References}

[1] Bliss, G. A. (1930) "An integral inequality", J. London Math. Soc. 5, 40-46.

[2] Carleman, T. (1923) "Sur les fonctions quasi-analytiques", Conferences faites au cinquieme congres des mathematiciens Scandinaves, Helsingfors, pp. 181-196.

[3] Cochran, J. A. and Lee, C.-S. (1984) "Inequalities related to Hardy's and Heinig's", Math. Proc. Camb. Phil. Soc. 96, 1-7.

[4] Heinig, H. P. (1975) "Some extensions of Hardy's inequality", SIAM J. Math. Anal. 6, $698-713$. 
[5] Heinig, H. P. (1990) "Weighted inequalities in Fourier analysis", In: Krbec, M., et al. (Eds.), Nonlinear Analysis, Function Spaces and Applications, Vol. 4. Proceedings of the Spring School at Roudnice nad Labem, 1990, Teubner Texte 119, Leipzig, pp. 42-85.

[6] Jain, P., Persson, L. E. and Wedestig, A. (2000) "From Hardy to Carleman and general mean-type inequalities", Function Spaces and Applications (Narosa Publ. House, New Delhi), pp. 117--130.

[7] Jain, P., Persson, L. E. and Wedestig, A. "Carleman-Knopp type inequalities via Hardy inequalities", Math. Ineq. Appl. 4 (2001), 343-355.

[8] Knopp, K. (1928) “Uber reihen mit positiven Gliedern", J. London Math. Soc. 3, 205211.

[9] Love, E. R. (1986) "Inequalities related to those of Hardy and of Cochran and Lee", Math. Proc. Camb. Phil. Soc. 99, 395-408.

[10] Manakov, V. M. (1992) "On the best constant in weighted inequalities for RiemannLiouville integrals", Bull. London Math. Soc. 24, 442-448.

[11] Opic, B. and Gurka, P. (1994) "Weighted inequalities for geometric means", Proc. Amer. Math. Soc. 120, 771-779.

[12] Pick, L. and Opic, B. (1994) "On geometric mean operator", J. Math. Anal. Appl. 183, $652-662$.

[13] Sinnamon, G. and Stepanov, V. D. (1996) "The weighted Hardy inequality: new proofs and the case $p=1$ ", J. London Math. Soc. 54(2), 89-101.

[14] Stepanov, V. D. (1994) "Weighted norm inequalities of Hardy type for a class of integral operators", J. London Math. Soc. 50(2), 105-120. 